\title{
ANALYSIS OF CALIBRATED SEA CLUTTER AND BOAT REFLECTIVITY DATA AT C- AND X-BAND IN SOUTH AFRICAN COASTAL WATERS
}

\author{
P.L. Herselman ${ }^{*}$, C.J. Baker ${ }^{\dagger}$ \\ ${ }^{*}$ Council for Scientific and Industrial Research, South Africa, <paul.herselman@ieee.org>, Fax: +27 (0)12 8412455 \\ ${ }^{\dagger}$ University College London, United Kingdom, <c.baker@ee.ucl.ac.uk>
}

Keywords: sea clutter; small boat; model; adaptive detection.

\begin{abstract}
The temporal characteristics of low grazing angle sea clutter and boat reflectivity are considered for both fixed and stepped frequency waveforms under a range of environmental conditions and geometrical configurations. Detectability of boats using an asymptotically optimal detector is evaluated empirically, as well as the influence of the local sea on boat reflectivity. Measurements were conducted with a calibrated, coherent, staring, pulsed radar system at $\mathrm{C}$ - and $\mathrm{X}$-band frequencies ranging from $6.9 \mathrm{GHz}$ to $10.3 \mathrm{GHz}$.
\end{abstract}

\section{Introduction}

Detection of small entities in sea clutter has been an active area of research in the international radar community for a continued period of time [5]. Persistent surveillance has become a top priority, with the plenary session at the 2005 IEEE International Radar Conference dedicated to persistent global area surveillance using space-based radar. In line with this, the AwareNet research theme [8] aims at the development of a persistent, ubiquitous maritime surveillance system with radar as the primary sensor. Typical entities of interest range from small recreational watercraft to large tanker ships. For small entities this will require a detector capable of discerning its minute reflection from the sea clutter returns for all environmental conditions and geometries over long distances.

Various models for modelling the average sea clutter reflectivity [10], amplitude statistics [11] [3], temporal correlation [3] as well as spatial correlation [1] have been proposed and validated. Typical scanning surveillance systems have to declare a detection from a limited number of pulses only. Due to the long decorrelation time of sea clutter [3] and the more often than not spiky amplitude statistics [11], detection is quite difficult due to the short dwell time. One of the characteristics of the envisaged AwareNet radar sensor will be its ability to employ long dwell times at specific areas of interest through the utilization of multiple, electronically steered receiver channels.

A sea clutter and boat reflectivity measurement trial was conducted from 25 July -4 August 2006 at the Overberg Test Range (OTB) on the south western coast of South Africa with the Council for Scientific and Industrial Research's (CSIR) Fynmeet dynamic Radar Cross Section (RCS) measurement facility (Figure 1). The aim of this trial was firstly to record datasets of sea clutter returns at different frequencies, range resolutions, grazing angles, look angles and environmental conditions to validate the state-of-the-art sea clutter models on South African coastal seawaters. Secondly, the aim was to record boat reflectivity datasets for a number of small boats to investigate its detectability with state-of-the-art detectors. This will lead to the development of improved detection algorithms for radar systems employing adaptive dwell times.

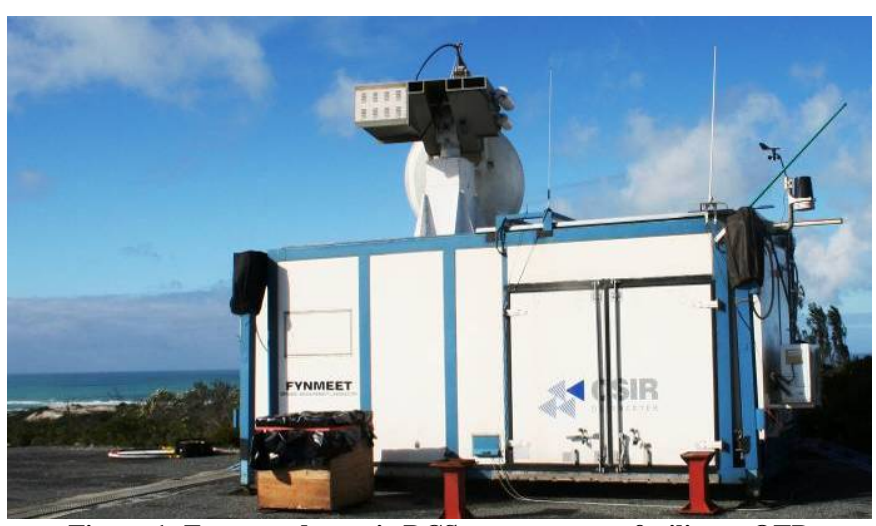

Figure 1: Fynmeet dynamic RCS measurement facility at OTB

Section 2 describes the Fynmeet radar and experimental setup, as well as the system and data integrity verification procedures. An overview of the measurement trial is presented in Section 3. Section 4 investigates a subset of the sea clutter measurements, focusing on the amplitude statistics and temporal characteristics for fixed frequency and frequency agile waveforms. Section 5 culminates with the analysis of various boat reflectivity measurements, investigating temporal correlation, dependence on the local sea, as well as the performance of the Adaptive Linear Quadratic (ALQ) Asymptotically Optimal Detector (AOD) [6] on small boats.

\section{Measurement Trial Description}

\subsection{Fynmeet Radar and Experimental Set-Up}

The important specifications of the Fynmeet mobile RCS measurement facility are listed in Table 1. 
Table 1: Fynmeet system and performance specifications

\begin{tabular}{|c|c|c|}
\hline \multirow{4}{*}{ 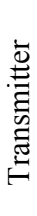 } & Frequency range & $6.5-17.5 \mathrm{GHz}$ \\
\hline & Peak power & $2 \mathrm{~kW}$ \\
\hline & PRF range & $0-30 \mathrm{kHz}$ \\
\hline & Waveforms & $\begin{array}{l}100 \& 300 \mathrm{~ns} \text { pulsed Continuous Wave } \\
(\mathrm{CW}) \text {, fixed / pulse-to-pulse frequency } \\
\text { agile }(500 \mathrm{MHz})\end{array}$ \\
\hline \multirow{4}{*}{ 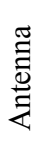 } & Type & Dual-offset reflector \\
\hline & Gain & $\geq 30 \mathrm{~dB}$ \\
\hline & Beam width & $\leq 2^{\circ}(3 \mathrm{~dB}$ beamwidth $)$ \\
\hline & Side lobes & $\leq-25 \mathrm{~dB}$ \\
\hline \multirow{5}{*}{ 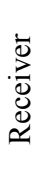 } & Dynamic range & $60 \mathrm{~dB}$ (instantaneous) / $120 \mathrm{~dB}$ (total) \\
\hline & Capture range & $200 \mathrm{~m}-15 \mathrm{~km}$ \\
\hline & Range gates & $1-64 ; \Delta \mathrm{R}=15 \mathrm{~m}$ or $45 \mathrm{~m}$ \\
\hline & Sampler type & I/Q Intermediate frequency sampler \\
\hline & Image rejection & $\leq-41 \mathrm{dBc}$ \\
\hline
\end{tabular}

The radar was deployed at OTB at location $34^{\circ} 36^{\prime} 56.52^{\prime \prime} \mathrm{S}$, $20^{\circ} 17^{\prime} 17.46^{\prime \prime} \mathrm{E}, 67 \mathrm{~m}$ above mean sea level. The shortest distance to the coastline was $1.2 \mathrm{~km}$ due south. A plan overview of the deployment site is depicted in Figure 2.

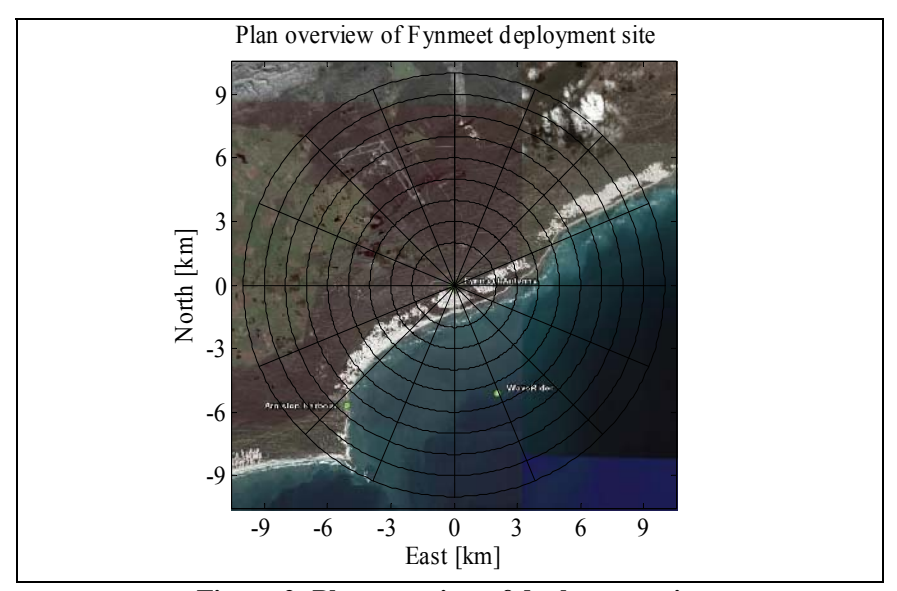

Figure 2: Plan overview of deployment site

Local wind speed and direction were logged with two weather stations separated by $1 \mathrm{~km}$. The local wave direction, significant wave height, maximum wave height and wave period were logged with a Datawell Directional Waverider Mark II wave buoy [2]. The local wave structure is influenced greatly by significant weather patterns from the south west and further perturbed by the diffraction patterns due to the cape, southwest of the deployment site which is located in a small bay area with a sea bed depth varying between $10-30$ $\mathrm{m}$ at ranges of 3 and $10 \mathrm{~km}$ respectively. The ground truth tracks of the boats were estimated using a differential processing Global Positioning System (GPS) receiver.

\subsection{System and Data Integrity Verification}

This section describes the measurements done and procedures followed to verify system and data integrity.

For absolute RCS calibration the response from a sphere suspended below a helicopter, tracked in range with a typical $\alpha-\beta$ tracker and in angle with a video centroid tracker, was measured and the calibration coefficient was calculated as

$$
C_{c a l}[d B]=20 \log 10\left(N^{-1} \sum_{n=1}^{N}\left|\sum_{m=M_{\max }(n)-1}^{M_{\text {max }}(n)+1} x(n, m)\right| A R^{4} / \sigma_{c s}\right),
$$

where $N$ is the number of pulses transmitted, $M_{\max }(n)$ the maximum range gate for the $n^{\text {th }}$ pulse, $A$ the receiver attenuation and $\sigma_{c s}$ the sphere RCS. A standard deviation of $1 \mathrm{~dB}$ was achieved. Daily stability verification measurements were done with a corner reflector, exhibiting variations in the order of about $1 \mathrm{~dB}$ across the measurement period.

Linearity of the quadrature receiver channels were ascertained by the analysis of calibrated noise source, receiver noise and blue sky measurements taken throughout the trial, analyzing channel skewness, kurtosis as well as the $2^{\text {nd }}$ to $4^{\text {th }}$ normalized intensity moments. The amplitude and phase imbalance of the quadrature channels were estimated as $0.03 \mathrm{~dB}$ and $\pm 1^{\circ}$ resulting in a negative Doppler image of $\leq-41 \mathrm{dBc}$. Additionally, there were also harmonically related spurious responses at a level of $\leq-50 \mathrm{dBc}$. A $5 \mathrm{MHz}$ leak-through signal was identified and removed from the data. The signal phase was non-deterministic and therefore the amplitude and phase were estimated from the dataset itself. Sea clutter with a strong steady state component biases this estimate and best results were obtained using a censored mean level technique in the estimation process. The percentage of the dataset used in the estimate yielding the lowest variance was chosen. Applying the Discrete Fourier Transform (DFT) to the corrected data, a $0 \mathrm{~Hz}$ frequency bin with a comparable power density to adjacent frequency bins was obtained without suppressing the steady state clutter response.

\section{Measurement Trial Overview}

Sea clutter datasets were recorded over a period of 11 days, with 112 fixed frequency and 38 stepped frequency datasets centred at four Transmit (Tx) frequencies over an azimuth angle range from $90^{\circ} \mathrm{N}$ to $225^{\circ} \mathrm{N}$ and a grazing angle range from $3^{\circ}$ to $0.3^{\circ}$. The local weather pattern may be described as roughly following a 6-day cycle [7] as cold front systems pass by from the west to the east. Over the trial period, the average wind speed ranged from $1 \mathrm{kt}$ to $20 \mathrm{kts}$, with a maximum gust of $40 \mathrm{kts}$. Wind direction spanned $360^{\circ}$, but the high wind speeds were mainly from the south west. The significant wave height varied between $1 \mathrm{~m}$ and $3.8 \mathrm{~m}$, with a maximum recorded wave height of $7.31 \mathrm{~m}$. The wave direction was roughly $180^{\circ} \mathrm{N}$, and slowly changed direction towards the end of the trial to $135^{\circ} \mathrm{N}$. Even with a low wind speed of $1 \mathrm{kt}$, the significant wave height was $\geq 1 \mathrm{~m}$. This is due to the strong incoming south westerly swell combined with the diffraction patterns of the close-by cape and the reduction in sea depth. The illuminated sea area can therefore not be defined as a fully developed sea [9] and the standard tables relating wind speed and wave height to sea state do not apply. In terms of the average wind speed, sea states 1 to a low 5 were observed. In terms of the wave height, sea states from a high 2 to a high 5 were observed. 


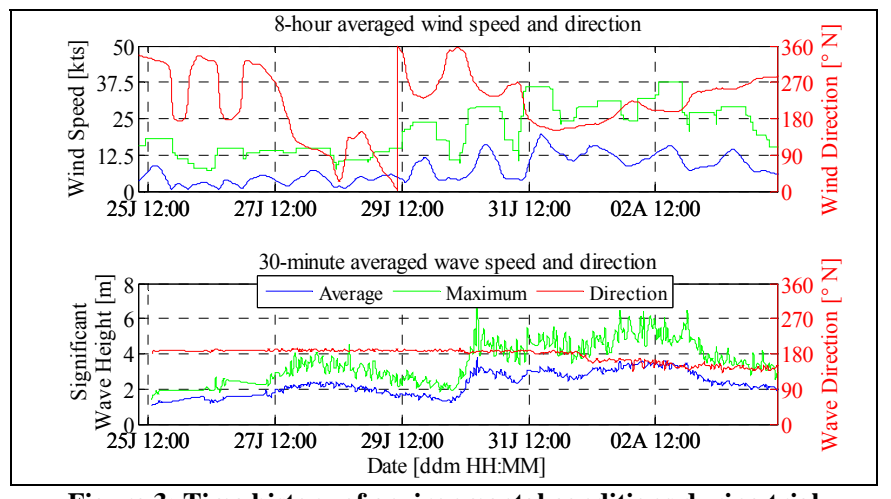

Figure 3: Time history of environmental conditions during trial

A Rigid Inflatable Boat (RIB), a glass fibre speed boat and a wooden chokka fishing vessel were deployed on four days with conditions ranging from calm to rough seas. The boats sailed a number of manoeuvres at different ranges and azimuth angles. 55 fixed frequency and 43 stepped frequency datasets were recorded centred at four $\mathrm{Tx}$ frequencies.

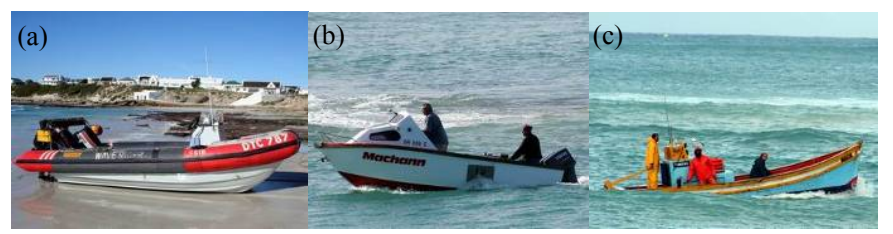

Figure 4: (a) RIB; (b) speed boat; (c) chokka fishing vessel

\section{Analysis of Sea Clutter Characteristics}

Various statistical properties are evaluated in this section for seven sea clutter datasets. These datasets represent low and high sea states at grazing angles $1^{\circ}$ and $0.5^{\circ}$ for a single Tx frequency and pulse widths of $100 \mathrm{~ns}$ and $300 \mathrm{~ns}$. The rangetime intensity plot and spectrogram for the high sea state, $1^{\circ}$, 100 ns dataset CFC16-001 are presented in Figure 5.

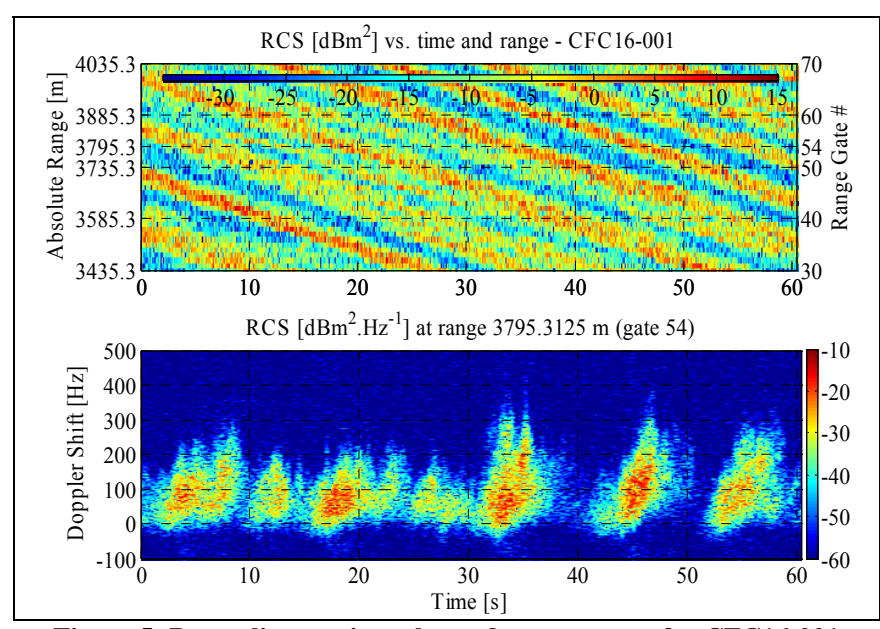

Figure 5: Range line vs. time plot and spectrogram for CFC16-001

The mean reflectivity and Clutter-to-Noise Ratio (CNR) for the different datasets are tabulated in Table 2 and compared to the Georgia Institute of Technology (GIT) and the Hybrid (HYB) model [10]. The $2^{\text {nd }}$ to $4^{\text {th }}$ normalised intensity moments and the estimated shape parameter $v$ are tabulated in Table 3 , assuming a $k$-distributed amplitude process and compared to the empirical shape parameter model [11].
Table 2: Empirical reflectivity $\left[\mathrm{dBm}^{2} / \mathrm{m}^{2}\right]$ and CNR [dB]

\begin{tabular}{|lllllll|}
\hline Sea State & Low & Low & Low & Low & High & High \\
Grazing Angle & $1^{\circ}$ & $0.5^{\circ}$ & $1^{\circ}$ & $0.5^{\circ}$ & $1^{\circ}$ & $0.5^{\circ}$ \\
Resolution [m] & 15 & 15 & 45 & 45 & 15 & 15 \\
CNR & 11 & -3 & 17 & 5 & 21 & 7 \\
Reflectivity & -48 & -51 & -51 & -52 & -39 & -41 \\
GIT model & -68 & -82 & -68 & -82 & -35 & -39 \\
HYB model & -42 & -48 & -42 & -48 & -36 & -39 \\
\hline
\end{tabular}

Table 3: Empirical vs. model amplitude statistics

\begin{tabular}{lllll|} 
Sea State & Low & Low & High & High \\
Grazing Angle & $1^{\circ}$ & $0.5^{\circ}$ & $1^{\circ}$ & $0.5^{\circ}$ \\
$M_{2}$ & 3.57 & 2.27 & 6.05 & 4.61 \\
$M_{3}$ & 24.88 & 9.47 & 72.32 & 63.75 \\
$M_{4}$ & 261.9 & 66.88 & 1238.6 & 1629.6 \\
$v$ - empirical & 1.18 & 2 & 0.57 & 1.22 \\
$v$ - model & 1.88 & 1.79 & 0.92 & 0.88 \\
\hline
\end{tabular}

The speckle sea clutter autocorrelation is plotted in Figure 6 for the different datasets. Its magnitude suggests correlation times between 10 and $20 \mathrm{~ms}$ that is related to sea state and grazing angle. The real and imaginary components reveal information regarding the mean Doppler shift, which is related to the wind and wave speeds and directions.

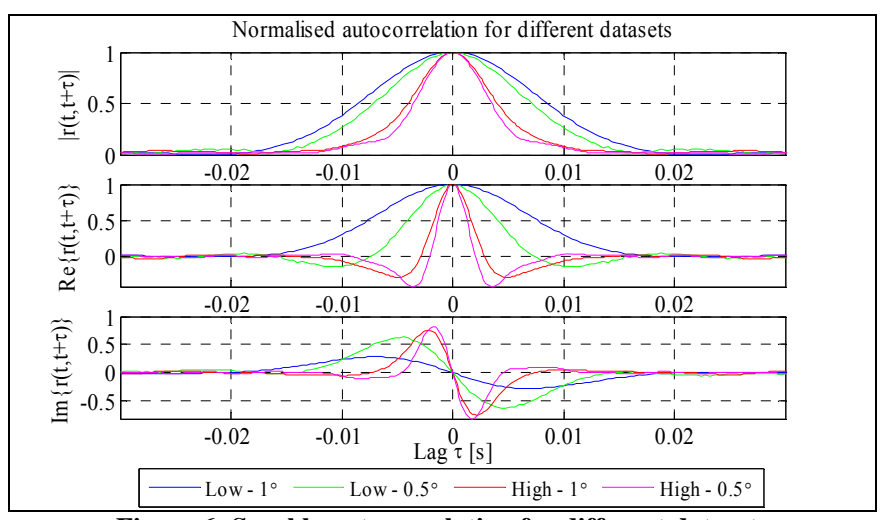

Figure 6: Speckle autocorrelation for different datasets

The pulse-to-pulse frequency agile cross correlation has also been evaluated and confirms that decorrelation occurs for frequency step sizes larger than the pulse bandwidth, except for discrete events (e.g. spikes) that exhibit significant cross correlation. The results in this section suggest that current empirical models accurately models sea clutter at the deployment site, except for the GIT model's overoptimistic prediction at low grazing angles and low sea states.

\section{Analysis of Small Boat Reflectivity}

\subsection{Fixed and Agile Frequency Temporal Characteristics}

In this section the temporal characteristics of small boats are evaluated. Isolation of the boat signature from the sea clutter was obtained by Doppler filtering, using GPS-estimated Doppler frequency as input. The responses of the three range gates closest to GPS range were coherently added to counter range-gate straddling. The resultant RCS time history of the chokka fishing boat is plotted in Figure 7. 


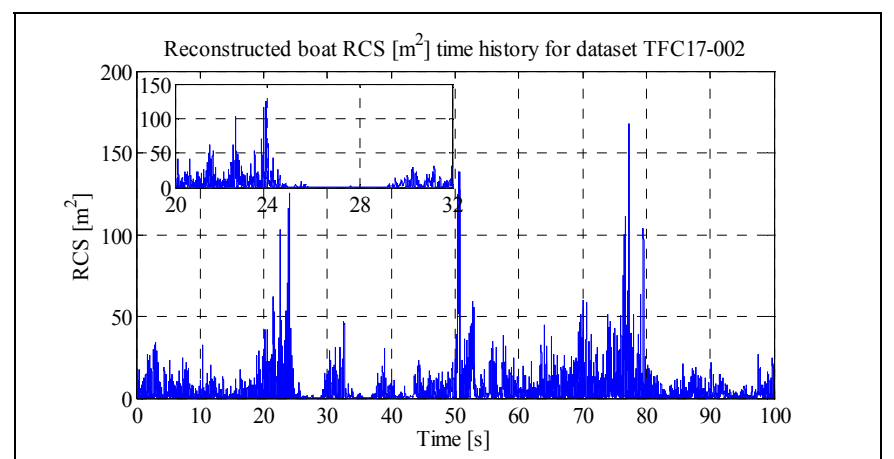

Figure 7: Reconstructed chokka boat RCS time history

The boat was steering a figure of eight with RCS peaks at 20 and $80 \mathrm{~s}$ associated with the starboard view and the peak at 50 $\mathrm{s}$ associated with the aft view. A characteristic of small boats in heavy sea is the fading of the RCS as the boat steers into the troughs of the waves, as indicated by the close-up view about 25 to $29 \mathrm{~s}$. This suggest strong correlation of boat RCS with the local sea waves. The non-coherent autocorrelation of a boat steering directly into the waves (Figure 8) shows periodicity with the same period as the mean sea wave period.

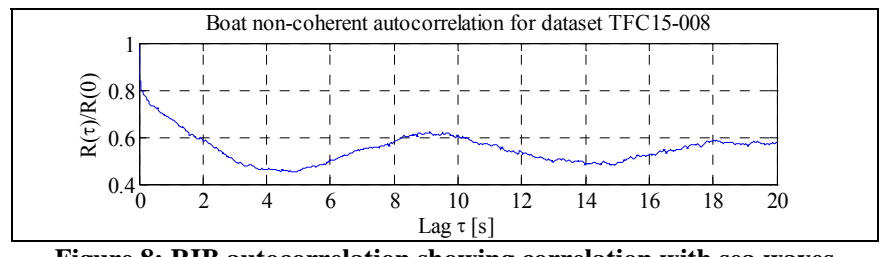

Figure 8: RIB autocorrelation showing correlation with sea waves

The empirical RCS Probability Density Function (PDF) of the RIB and the fishing vessel are compared with the standard Swerling models 1 and 3 [9] in Figure 9. Slight difference between the PDF's of the two boats are visible as well as the inability of the Swerling models to accurately describe their amplitude distributions.

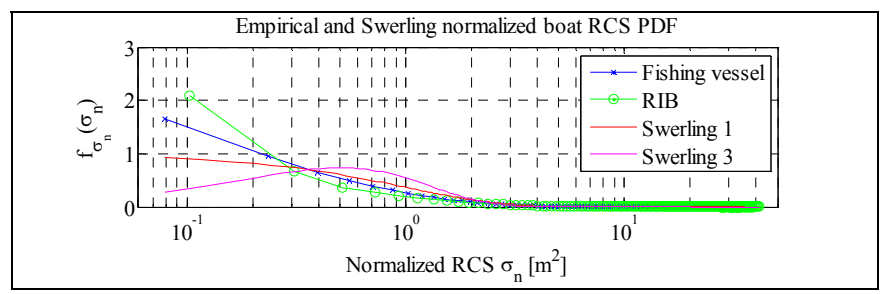

Figure 9: RIB and fishing vessel RCS PDF

The optimal Coherent Processing Interval (CPI) was estimated for the different boats from their Doppler response by identifying the CPI where the boat response spreads over multiple Doppler cells.

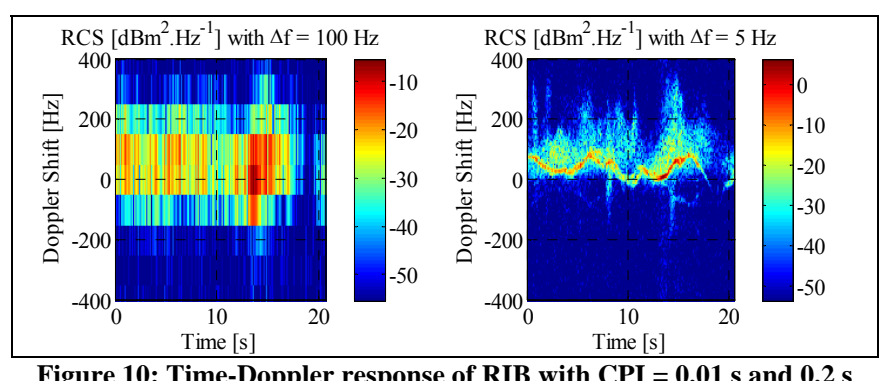

Figure 10: Time-Doppler response of RIB with CPI $=0.01 \mathrm{~s}$ and $0.2 \mathrm{~s}$
For all boats in most datasets this occurred at CPI's exceeding $0.2 \mathrm{~s}$. The significant gain in increasing the CPI is evident in Figure 10, where the boat response can be discerned from the sea clutter.

The frequency agile pulse-to-pulse correlation coefficient

$$
\rho_{n m}=C_{n m} / \sigma_{n} \sigma_{m}=E\left\{\left(\mathbf{x}_{n}-\eta_{n}\right)\left(\mathbf{x}_{m}^{*}-\eta_{m}^{*}\right)\right\} / \sigma_{n} \sigma_{m},
$$

where $\mathbf{x}_{k}$ is the return vector for the $k^{\text {th }}$ Tx frequency in the stepped frequency waveform, was calculated for range gates containing sea clutter only and range gates containing both sea clutter and boat reflectivity. The results for the chokka fishing boat are plotted in Figure 11. Pulse-to-pulse decorrelation was observed for sea clutter only, with significant levels of correlation exhibited for range gates containing both sea clutter and boat reflectivity.

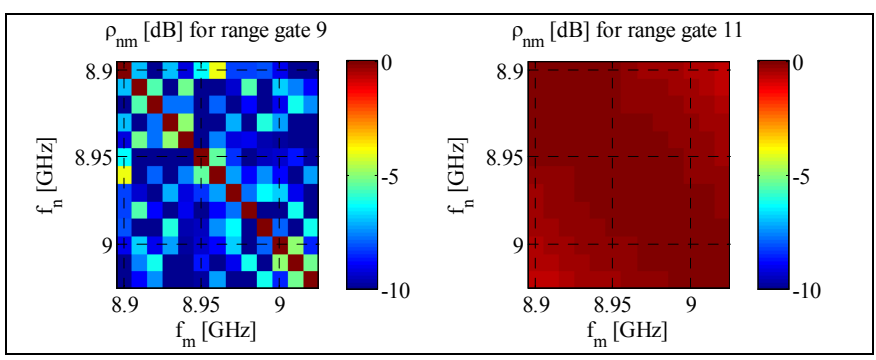

Figure 11: Correlation coefficient for sea clutter and fishing vessel

The results in this section provide useful insights into small boat characteristics and how it deviates from basic simulation models where the interaction between the sea and boats are disregarded, highlighting the need to incorporate these effects in realistic numerical simulation models.

\subsection{Detectability of Real-Life Small Boats}

Various detectors have been proposed for the detection of targets in sea clutter, including the AOD ALQ detector [4] which has mainly been evaluated on real sea clutter data with superimposed Swerling 1 to 4 targets. In this section, the performance of the ALQ detector is evaluated on datasets with real-life boats. The ALQ detector [6] estimates the covariance matrix $\mathbf{M}$ of the test cell from $K$ adjacent range bins over a CPI of $m$ pulses using the iterative equation

$$
\hat{\mathbf{M}}(i+1)=\frac{m}{K} \sum_{k=1}^{K} \frac{\mathbf{z}_{k} \mathbf{z}_{k}^{H}}{\mathbf{z}_{k}^{H} \hat{\mathbf{M}}^{-1}(i) \mathbf{z}_{k}} ; \hat{\mathbf{M}}(0)=\mathbf{I},
$$

where $\mathbf{z}_{k}$ is the $k^{\text {th }}$ range gate complex radar return vector. The detector employs the generalized likelihood ratio test

$$
\frac{\left|\mathbf{p}^{H} \mathbf{M}^{-1}\right|^{2}}{\left(\mathbf{p}^{H} \mathbf{M}^{-1} \mathbf{p}\right)\left(\mathbf{z}^{H} \mathbf{M}^{-1} \mathbf{z}\right)} \stackrel{H_{1}}{<}, \lambda,
$$

where $\mathbf{p}$ is the boat steering vector. The detector was evaluated with $K=3 m$, a single range guard cell and the number of iteration in (3) equal to $\mathrm{N}_{\mathrm{it}}=3$. The results for a RIB dataset with a $-6.3 \mathrm{~dB}$ average Signal-to-Clutter Ratio 
(SCR) are depicted in Figure 12, yielding an average $\mathrm{P}_{\mathrm{d}}$ of $13.8 \%$.

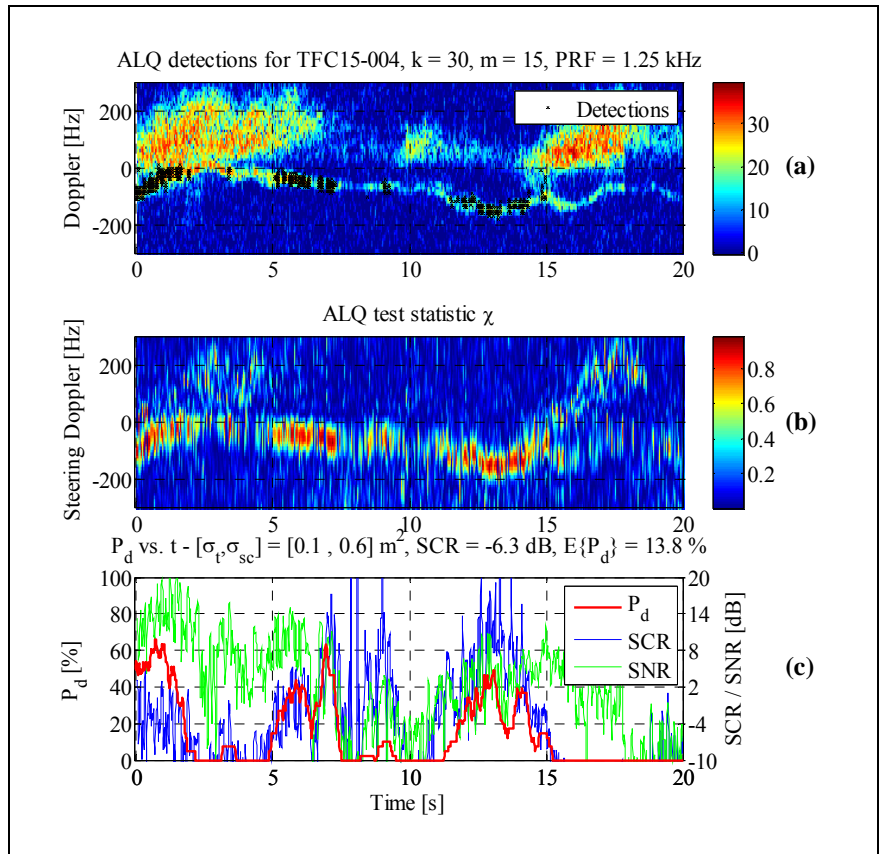

Figure 12: ALQ detection performance for RIB dataset TFC15-004:

(a) time-Doppler response with detections overlay, (b) ALQ test statistic, (c) time history of $P_{d}$, SCR and SNR

Decorrelation of the sea clutter with an average Doppler spread is evident, as is the inability to effectively decorrelate events with a significantly different Doppler spread, e.g. whitecaps or a discrete spike. The boat Doppler energy is smeared by the whitening filter, but still yields high levels of $\mathrm{P}_{\mathrm{d}}$ for short periods of time, typically when the SNR is high and/or there is ample Doppler separation, even though the $\mathrm{SCR}$ is quite low (e.g. $0 \leq \mathrm{t} \leq 2 \mathrm{~s}$ ). For an injected Swerling 1 target with the same SCR at $2.5 \mathrm{kts}$, the average $\mathrm{P}_{\mathrm{d}}=11.4 \%$ with a more even distribution of detections over time. In larger SCR datasets, e.g. the fishing vessel in dataset TFA17-001 with SCR $=6.6 \mathrm{~dB}, \mathrm{P}_{\mathrm{d}}$ increased to $55.4 \%$ with regular intervals where $P_{d} \geq 80 \%$. Qualitative analysis suggests possible improvement by reducing the reference window in the range dimension and extending it in the time dimension, i.e. $K<<3 m$, since this may result in a better estimate of the local temporal correlation of the sea.

\section{Conclusions}

A comprehensive set of sea clutter and boat reflectivity datasets was recorded at $\mathrm{C}$ - and $\mathrm{X}$-band for low grazing angles in the South African coastal waters. The datasets were used to verify current sea clutter models, to investigate the characteristics of small boat reflectivity and to gain an appreciation of the performance of AOD algorithms on real targets. From the insights gained possible improvements were suggested. Of particular interest were the dependency of the boat reflectivity on the local sea, deviation from the Swerling RCS models, the perceived persistence of reflectivity for short periods of time and the distinguishable pulse-to-pulse frequency agile correlation properties of small boats.

\section{Acknowledgements}

The authors acknowledge the CSIR team for their contribution in the planning and execution of the trial; as well as the Armaments Corporation of South Africa and the South African Air Force for permission to use the Fynmeet radar. The research was co-funded by the South African Department of Science and Technology, the South African Department of Defence and the Royal Society.

\section{References}

[1] B.C. Armstrong \& H.D. Griffiths. 'Modelling spatially correlated K-distributed clutter', Electronic Letters 27(15), pp. 1355 - 1356, 1991.

[2] 'Datawell BV oceanographic instruments', [Accessed 28th November 2006], Available from World Wide Web: $<$ http://www.datawell.nl/inhoud.php?id=3>, 2006.

[3] A. Farina; F. Gini; M.V. Greco \& L. Verrazzani. 'High resolution sea clutter data: statistical analysis of recorded live data', IEE proc., radar sonar navig. 144(3), pp. 121 $-130,1997$.

[4] F. Gini; M. Greco; M. Diani \& L. Verrazzani. 'Performance analysis of two adaptive radar detectors against non-Gaussian real sea clutter data', IEEE T-AES 36(4), pp. 1429 - 1439, 2000.

[5] F. Gini; A. Farina \& M. Greco. 'Selected list of references on non-gaussian radar detection', IEEE T-AES 37(1), pp. 329 - 359, January 2001.

[6] F. Gini \& M. Greco. 'Covariance matrix estimation for CFAR detection in correlated heavy tailed clutter', Signal Processing 82(12), pp. 1847 - 1859, 2002.

[7] R. Hamann. 'Wind climatology of the South Western Cape', [Accessed 28 November 2006], Available from World Wide Web: <http://www.windsurfingafrica. org/Articles/tabid/285/ctl/ArticleView/mid/676/articleId/ 5/Wind-climatology-of-the-South-Western-Cape--byRalph-Hamann.aspx>, 2006.

[8] M. Hill. 'South Africa flying high - or just pie in the sky: Maritime area-surveillance proposal could become national flagship project', Engineering News 26(6), pp. 38 - 39, 24 February - 3 March 2006.

[9] F.E. Nathanson. Radar Design Principles: Signal Processing and the Environment, SciTech Publishing, Inc., Mendham NJ, 1999.

[10]J.P. Reilly \& G.D. Dockery. 'Influence of evaporation ducts on radar sea return', IEE Proceedings F 137(2), pp. $80-88,1990$.

[11]K.D. Ward; C.J. Baker \& S. Watts. 'Maritime surveillance radar, Part 1: Radar scattering from the ocean surface', IEE Proceedings F 137(2), pp. 51 - 62, 1990. 